\section{Utilização das informações vitais para a estimação de indicadores de mortalidade no Brasil: da busca ativa de eventos ao desenvolvimento de métodos}

\section{Use of vital data to estimate mortality indicators in Brazil: from the active search for events to the development of methods}

\section{Utilización de la información vital para la estimación de indicadores de mortalidad en Brasil: de la búsqueda activa de eventos al desarrollo de métodos}

\section{Resumo}

O artigo apresenta a construção histórica brasileira sobre a utilização das informações vitais, incorporando procedimentos de avaliação das informações e pesquisas de busca ativa de nascimentos e óbitos, que resultaram na proposição de métodos para o cálculo de indicadores de natalidade e mortalidade mediante o uso de registros contínuos. Além das pesquisas para captar eventos vitais referentes aos anos de 2000 e 2008, são apresentados os procedimentos para a correção dos eventos informados aos sistemas de informação e a mudança de paradigma no método de cálculo dos indicadores de mortalidade decorrente destas iniciativas. Adicionalmente, destacam-se os avanços na adequação das informações sobre óbitos e nascidos vivos no Brasil, as alterações nas estimativas da mortalidade infantil decorrentes da proposição de métodos, além do desafio de estimar o indicador para áreas geográficas subnacionais, com menores contingentes populacionais, que em sua maioria é composta por municípios com baixa cobertura e regularidade dos dados.

Estatísticas Vitais; Sistemas de Informação; Registros de Mortalidade;

Registro de Nascimento
Paulo Germano de Frias 1

Célia Landmann Szwarcwald 2

Otaliba Libânio de Morais Neto 3

Maria do Carmo Leal 4

Juan José Cortez-Escalante 5

Paulo Roberto Borges de Souza Junior 2

Wanessa da Silva de Almeida 2

Jarbas Barbosa da Silva Junior 6

doi: 10.1590/0102-311X00206015

\section{Correspondência}

P. G. Frias

Instituto de Medicina Integral Prof. Fernando Figueira.

Rua dos Coelhos s/n, Recife, PE 50070-590, Brasil.

pfrias@imip.org.br

1 Instituto de Medicina Integral Prof. Fernando Figueira, Recife, Brasil.

2 Instituto de Comunicação e Informação Científica e

Tecnológica em Saúde, Fundação Oswaldo Cruz, Rio de Janeiro, Brasil.

3 Instituto de Patologia Tropical e Saúde Pública, Universidade Federal de Goiás, Goiânia, Brasil.

${ }_{4}$ Escola Nacional de Saúde Pública Sergio Arouca, Fundação Oswaldo Cruz, Rio de Janeiro, Brasil.

5 Organização Pan-Americana da Saúde, Brasília, Brasil.

6 Agência Nacional de Vigilância Sanitária, Brasília, Brasil. 


\section{Introdução}

O ano de 2015 delimita o prazo para que os 190 países signatários do pacto com a Organização das Nações Unidas sobre os oito Objetivos de Desenvolvimento do Milênio (ODM) seja efetivado e retrate o compromisso social com a sobrevivência e direitos humanos inalienáveis, em especial de grupos vulneráveis como mulheres e crianças ${ }^{1}$. Para monitorar o cumprimento de tal desafio, foi necessário adotar uma multiplicidade de abordagens metodológicas que considerasse a realidade nacional e a disponibilidade de informações válidas, acuradas e representativas do território nacional 2.

No Brasil, apesar da disponibilidade do Sistema de Informações sobre Mortalidade (SIM) e do Sistema de Informação sobre Nascidos Vivos (SINASC) para praticamente todo o período, as coberturas e qualidade dos dados desiguais entre as regiões e Unidades da Federação (UF) impunham limites quanto ao seu uso 3. Os dados vitais informados ao Ministério da Saúde são disponibilizados na Internet (http://www.datasus.gov.br), no nível de município, e as consultas são acessíveis pelos aplicativos Tabwin/Tabnet e por painéis situacionais de monitoramento, viabilizando a identificação de irregularidades locais de forma cada vez mais ágil 4.

Desde a criação dos dois sistemas de informações, o Estado brasileiro tem investido para o seu aperfeiçoamento, em particular na última década, obtendo em geral êxito expressivo 4,5. Embora persistam alguns municípios com precariedade dos dados em alguns estados, especialmente os localizados na Amazônia Legal e no Nordeste, optou-se, no Brasil, pela elaboração dos indicadores de natalidade e mortalidade, em âmbito nacional, por meio do uso dos dados vitais informados e corrigidos por fatores de correção estimados em pesquisas amostrais de busca ativa de óbitos (RIPSA - Rede Interagencial de Informações para a Saúde. Indicadores e dados básicos para a saúde, 2012. http:// tabnet.datasus.gov.br/cgi/idb2012/matriz.htm).

Em muitos países, diante da inexistência de sistemas de informação sobre eventos vitais de registro contínuo ou da disponibilidade de dados fidedignos, a utilização de técnicas demográficas indiretas constituiu uma alternativa para a análise da situação da mortalidade infantil no período 1990-2000 6,7. Em anos subsequentes, variantes do método foram aperfeiçoados e utilizados em vários países nas pesquisas sobre demografia e saúde 8 .

Se as técnicas demográficas baseadas em pesquisas por amostragem são uma alternativa para países com informações vitais precárias ou inexistentes, desvantagens são recorrentemente apresentadas. Entre elas, a principal é a de não ser baseada em registros administrativos contínuos, impossibilitando o monitoramento da situação e a avaliação de desempenho de intervenções. Ademais, dependem do tamanho da amostra, que, muitas vezes, não permite a estimação da mortalidade infantil em recortes geográficos adequados, como os municípios. Problemas na interpretação dos indicadores de saúde ocorrem, adicionalmente, porque os estimadores são tratados como parâmetros populacionais, sem estimação do erro das estimativas pontuais e sem consideração do efeito de desenho de amostragem 9,10 .

Contudo, no Brasil, o uso de técnicas demográficas para avaliar a cobertura do SIM funcionou como uma validação dos resultados encontrados por meio das pesquisas de busca de óbitos. Os achados foram muito proveitosos, sugerindo que os esforços governamentais para melhorar a qualidade dos dados foram bem-sucedidos 11 .

Considerando as limitações da técnica indireta e a melhoria sistemática do SIM e SINASC, aliada ao intenso debate internacional acerca das melhores alternativas metodológicas para monitorar a situação dos países quanto ao alcance dos ODM, o interesse nos sistemas de registro contínuo foi ampliado 12,13 .

Este manuscrito tem por objetivo apresentar a construção histórica brasileira sobre a utilização das informações vitais, incorporando procedimentos de avaliação da adequação das informações e pesquisas de busca ativa de nascimentos e óbitos, que resultaram na proposição de métodos para o cálculo de indicadores de natalidade e mortalidade mediante o uso de registros contínuos. 


\section{História das estimativas brasileiras de mortalidade}

Ao longo da década de 1990, diante da indisponibilidade de informações completas sobre nascidos vivos e óbitos para o país e UF, o Ministério da Saúde divulgava estimativas da mortalidade infantil elaborada por meio de técnicas indiretas pelo Instituto Brasileiro de Geografia e Estatística (IBGE) 14. No âmbito estadual, a cada divulgação dos dados, reações diversificadas eram observadas, relacionadas à contestação das estimativas, ampliação do acesso às políticas públicas para enfrentamento do problema, e à mobilização de recursos técnicos para definir estratégias para melhorar a captação dos eventos vitais 15,16 .

Apesar das iniciativas estaduais localizadas, apenas com a divulgação em 1999 de estimativas indiretas das taxas de mortalidade infantil para todos os municípios brasileiros com mais de 80 mil habitantes 17, incluindo os que dispunham de dados do SIM e SINASC com qualidade consagrada, intensificou-se o debate sobre a pertinência do uso de técnicas demográficas indiretas para todas as UF 10. As estimativas resultantes para os municípios com informações completas e de boa qualidade mostraram-se superestimadas e, adicionalmente, apresentaram inconsistências relevantes para alguns estados 18,19. De acordo com as estimativas do IBGE, havia flutuação negativa na cobertura do SIM de estados como Rio de Janeiro e São Paulo, recuando entre 1997 e 1998 de 109\% para 89\% e de $92 \%$ para $82 \%$, respectivamente, enquanto o Acre teria ampliado a cobertura do SIM de $56 \%$ para $82 \%$ em apenas um ano 18.

Considerando os investimentos direcionados à redução da mortalidade infantil das três instâncias federativas em curso, a exemplo do Projeto de Redução da Mortalidade Infantil, as taxas estimadas indiretamente repercutiram negativamente e, em decorrência, pesquisadores, gestores e profissionais de saúde céticos em relação ao seu uso escreveram manifesto dirigido ao Ministério da Saúde 10.

Em consonância com essa situação, em 2000, o Ministério da Saúde convocou especialistas para discutir estratégias que favorecessem a consolidação dos sistemas de informação vitais de registro contínuo em substituição às técnicas indiretas para o cálculo da mortalidade infantil. Algumas das alternativas apresentadas pelo grupo foram a análise da adequação das informações vitais para o uso adequado dos sistemas, bem como a realização de processos de busca ativa dos eventos vitais para captar casos não informados ao Ministério da Saúde. Em adição, foram sugeridos instrumentos que favorecessem a integração dos sistemas de informações do Ministério da Saúde, gerando a edição de cartilha para os profissionais de Saúde da Família, que abordava a integração SIM, SINASC e Sistema de Informação da Atenção Básica (SIAB) no âmbito local. Como primeiro resultado da discussão, a RIPSA passou a divulgar os Indicadores e Dados Básicos - Brasil 2000 (IDB-RIPSA. http://tabnet. datasus.gov.br/cgi/idb2000/matriz.htm), contendo estimativas calculadas com os dados vitais informados para as UF que tinham cobertura considerada completa e regularidade no envio das informações vitais (Espírito Santo, Rio de Janeiro, São Paulo, Paraná, Santa Catarina, Rio Grande do Sul, Mato Grosso do Sul e Distrito Federal).

O principal problema que se apresentava era responder objetivamente: Como estimar a mortalidade infantil e avaliar tendências em áreas com informações vitais precárias? Dando seguimento às propostas encaminhadas pelos especialistas na reunião com o Ministério da Saúde, Szwarcwald et al. 18 propuseram uma metodologia para avaliar a adequação das informações vitais, estabelecendo critérios para identificar municípios com limitações nas informações e um índice para sintetizar a adequação dos dados vitais por UF. A adequação foi analisada com base em indicadores para aferir a cobertura (coeficiente geral de mortalidade padronizada por idade e taxa de natalidade) e a regularidade (desvio médio relativo do coeficiente geral de mortalidade e desvio médio da taxa de natalidade) referentes ao SIM e SINASC, respectivamente. Para avaliar a qualidade do SIM observou-se também a proporção de óbitos de menores de um ano por causas mal definidas 18 .

Os municípios foram agregados em estratos de acordo com a categoria de adequação das informações e região. A Figura 1 apresenta os municípios das regiões Norte e Nordeste segundo nível de adequação das informações de óbitos no triênio 1996-1998. Chama a atenção o grande número de municípios com coeficiente geral de mortalidade padronizada por idade inferior a 2,00 por mil habitantes, com diferenciais importantes entre as regiões e UF, variando de 3,1\% na Região Sul a 38,5\% na Região Norte. 


\section{Figura 1}

Coeficiente geral de mortalidade padronizado por idade por municípios. Regiões Norte e Nordeste, Brasil, 1996-1998.

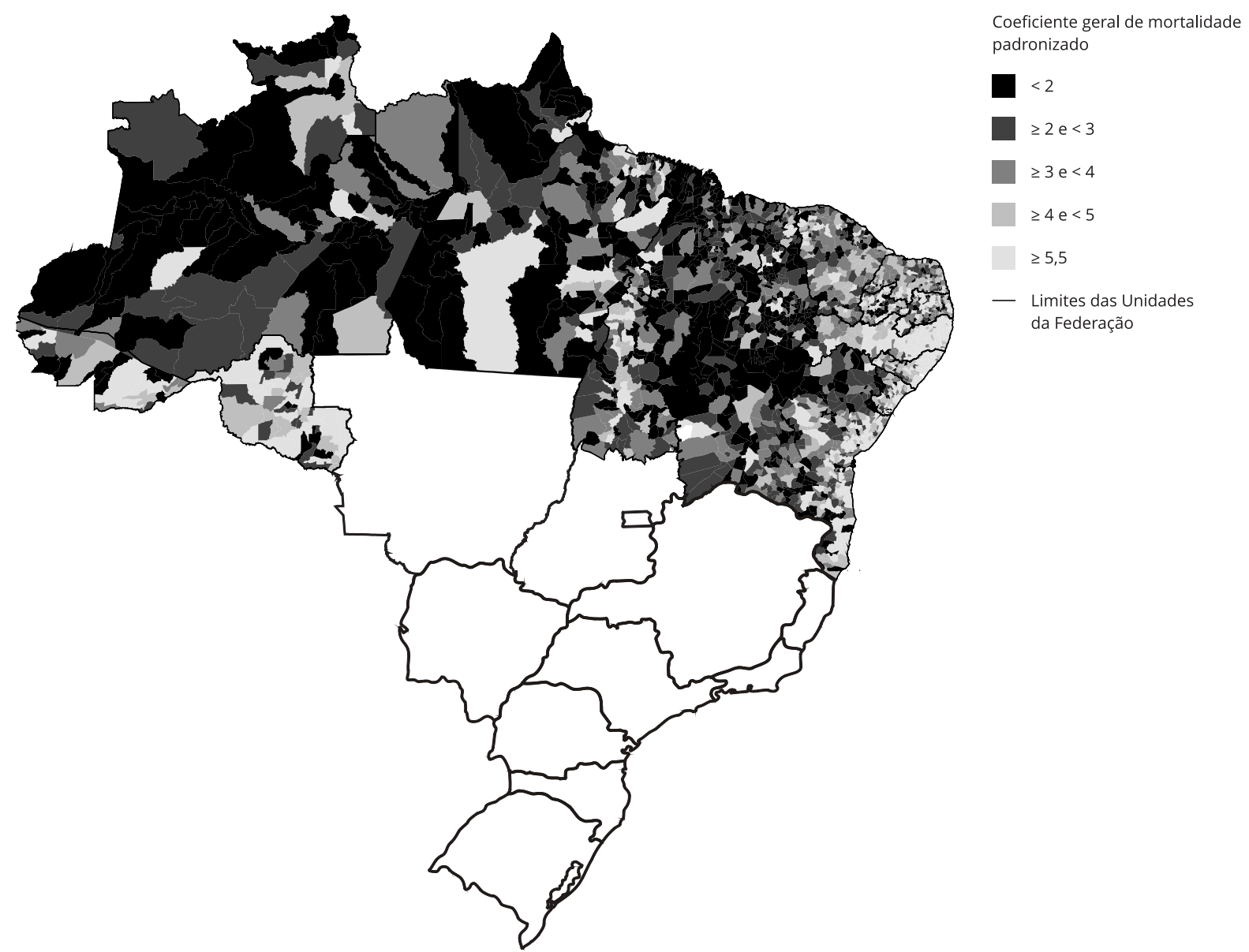

\section{A primeira busca ativa de óbitos}

Em continuidade às recomendações da reunião do Ministério da Saúde, foi organizada a primeira pesquisa de busca ativa em municípios brasileiros, intitulada Busca Ativa de Óbitos em Aglomerados de Municípios Brasileiros com Grande Precariedade das Informações Vitais, referente aos óbitos infantis ocorridos no ano 2000. Para alcançar um número suficientemente grande de óbitos que possibilitasse a estimação da mortalidade infantil, definiu-se que cada conglomerado (composto por municípios vizinhos com grande precariedade das informações vitais) teria pelo menos 50 mil habitantes. Outra condição era que esses municípios fossem cobertos pelo Programa Agentes Comunitários de Saúde e Programa Saúde da Família (PACS/PSF), para que as informações do SIAB pudessem ser validadas. Foram selecionados dois estados na Região Norte (Pará e Amazonas), e quatro na Região Nordeste (Maranhão, Piauí, Paraíba e Bahia), que apresentaram os piores índices de adequação das informações no período 1996-199820.

$\mathrm{Na}$ primeira pesquisa de busca ativa, para a coleta de dados utilizou-se um instrumento padronizado para a identificação tanto de Declarações de Óbito (DO) emitidas e não informadas ao SIM como de óbitos que não geraram as respectivas DO, compreendendo os óbitos fetais e não fetais de 
menores de um ano residentes nos municípios selecionados. Como fontes de informações foram usados registros obtidos em cartórios, cemitérios oficiais e não oficiais, funerárias, estabelecimentos de saúde, no PACS/PSF além de informantes-chave, parteiras tradicionais, líderes comunitários, curandeiros e rezadeiras. Foram utilizadas múltiplas fontes para avaliar a confiabilidade das informações coletadas 20 .

Além de possibilitar a estimação da mortalidade infantil, usando como base as coberturas estimadas de óbitos infantis nos municípios com grande precariedade das informações 18 , o processo de busca ativa de óbitos apontou falhas na operacionalização do SIM. Os problemas identificados se relacionaram à organização do sistema, em particular à notificação insuficiente, coleta irregular e fluxos inadequados entre as esferas de gestão 20.

A Figura 2 apresenta uma sepultura de um óbito infantil no quintal da residência de uma família cujo chefe tinha formação universitária, sugerindo a adoção de hábitos de enterramento de acordo com a cultura local, independentemente do grau de educação 21,22. Outra situação inesperada foi a identificação de declaração de óbito e de nascimento produzidas pelo nível local, diferentes do modelo original, que foram encontradas no processo de busca ativa e associadas, igualmente, aos aspectos culturais envolvidos na produção da informação e ao desconhecimento das normas legais instituídas. Embora estudos anteriores já tenham indicado a presença de enterramentos sem a declaração de óbito 23 , a identificação de produção local de documentos foi uma situação inédita evidenciada na pesquisa de busca ativa do ano 2000.

Até essa etapa, os avanços alcançados contemplaram a adoção pelo Ministério da Saúde dos critérios de adequação das informações de mortalidade para a definição de metas a serem atingidas pelas UF, a recomendação de medidas para integração de sistemas (SIAB, Sistema de Informações Hospita-

\section{Figura 2}

Sepultura em quintal de residência no interior do Nordeste, Brasil, casos identificados durante a busca ativa de óbitos e nascimentos.

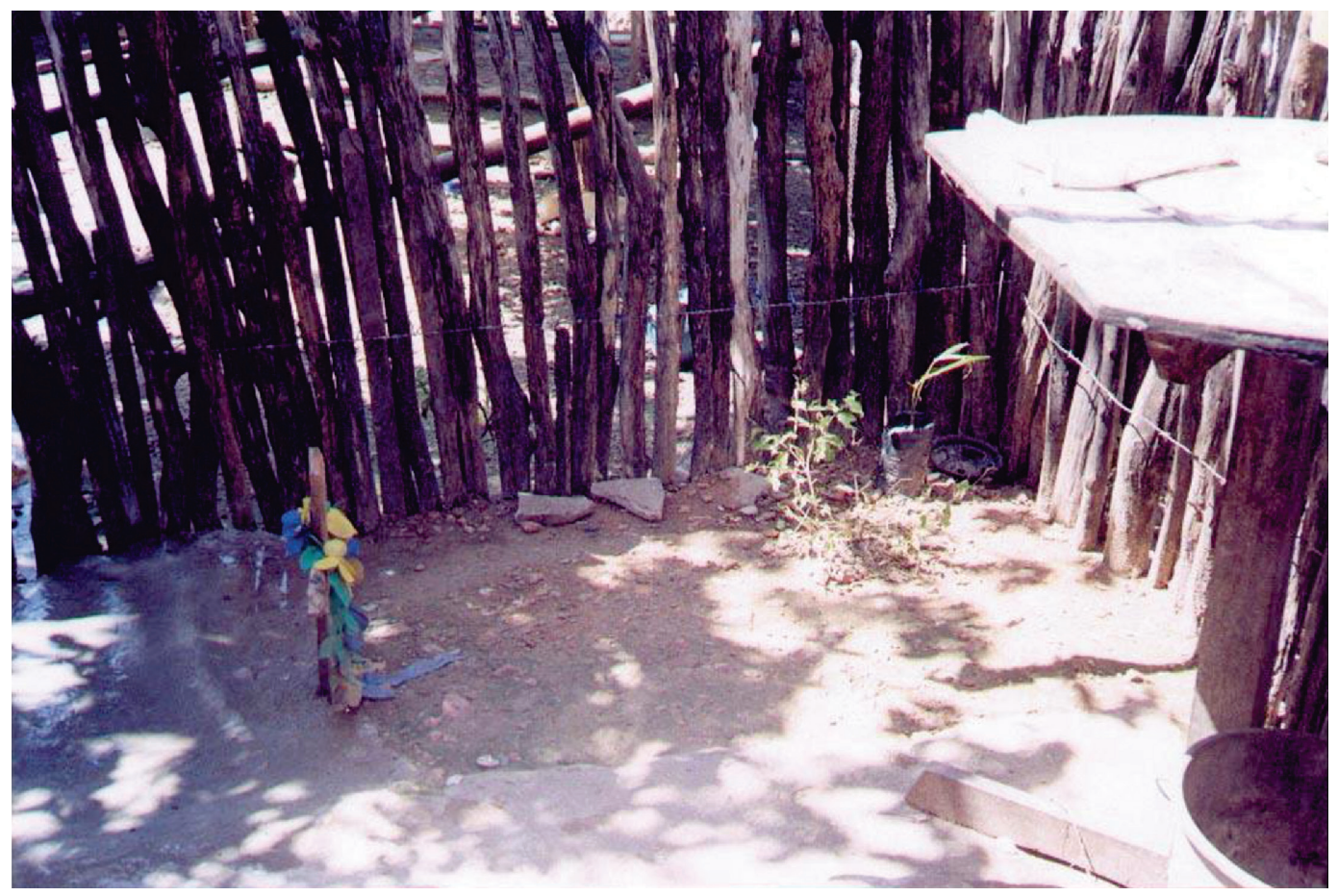


lares - SIH, Sistema de Informação de Agravos de Notificação - SINAN, SINASC e SIM) 24, a contratação de consultores locais para a melhoria da qualidade do preenchimento das DO, sobretudo a causa básica, e para a ampliação da cobertura dos dados vitais, incluindo a identificação e o cadastramento de cemitérios não oficiais 14,24,25. Concomitantemente, na medida em que foram utilizados pelos gestores e pesquisadores, os indicadores de avaliação das informações vitais foram aperfeiçoados 26 .

A análise do período compreendido entre os anos iniciais do processo de discussão em curso, 1996-1998 até 2005-2007, demonstrou os progressos para os cinco indicadores de adequação das informações vitais relacionados à cobertura, regularidade e proporção de óbitos com causa mal definida 10. Por sua vez, os municípios que integraram os conglomerados da busca ativa mantiveram, em geral, avanços na captação dos óbitos após alguns anos, indicando a relevância da integração dos profissionais de âmbito local nas atividades de captação de óbitos e a implantação dos processos de busca ativa na rotina do município 20.

Apesar dos progressos alcançados com a primeira busca ativa de óbitos infantis, as questões sobre como estimar indicadores de mortalidade no Brasil por meio das informações do SIM e do SINASC, e como avaliar, em curto prazo, as ações dirigidas a reduzir a mortalidade infantil, permaneciam sem uma solução robusta. O uso de métodos demográficos indiretos ainda era o procedimento adotado para as UF do Norte, Nordeste; Mato Grosso e Goiás, no Centro-oeste; e Minas Gerais, no Sudeste 10,20.

No contexto do plano de redução das desigualdades regionais do Nordeste e Amazônia Legal 2009-2010, coube ao Ministério da Saúde a meta de redução das desigualdades regionais da Mortalidade Infantil, que induziu o desenvolvimento de ações voltadas para a melhoria da cobertura e qualidade das informações de mortalidade relacionadas à redução das causas mal definidas e a ampliação da cobertura dos óbitos infantis. Além disso, diante da experiência exitosa do primeiro processo de busca ativa de eventos vitais, empreendeu-se a segunda pesquisa de busca ativa, como estratégia de definição de fatores de correção do sub-registro de óbitos e nascidos vivos, que possibilitassem a obtenção de estimativas mais adequadas da mortalidade infantil.

\section{Segunda busca ativa de óbitos e nascimentos na Amazônia Legal e no Nordeste}

Com o reconhecimento institucional das potencialidades do método e o financiamento tripartite, foi considerada uma amostra probabilística de mais de 133 municípios localizados em 16 UF no Nordeste e na Amazônia Legal para a captação de óbitos e nascimentos ocorridos em 2008 e não informados ao Ministério da Saúde 27.

A amostra foi estratificada por porte populacional, nível de adequação das informações vitais e região constituindo 24 estratos, selecionando-se pelo menos 4 municípios em cada estrato. Todas as capitais foram incluídas na pesquisa. O objetivo da investigação foi estimar as coberturas do SIM e Sinasc por estrato, para possibilitar a estimação de indicadores de mortalidade por UF, como a taxa geral de mortalidade e a mortalidade infantil 27,28 .

O trabalho de campo foi conduzido entre setembro de 2009 e junho de 2010. Nos municípios selecionados, foi realizado um processo de busca ativa de eventos vitais ocorridos no ano de 2008 , para identificar tanto as Declarações de Nascido Vivo (DNV) e de DO emitidas e não informadas ao SINASC e ao SIM, respectivamente, como os nascidos vivos e óbitos para os quais não foram emitidas as respectivas DNV e DO. Foram utilizadas as seguintes fontes de informações: cartórios; secretarias de ação social (Cadastro Único dos Programas Sociais do Governo Federal - Bolsa Família, auxílio sepultamento, auxílio enxoval); hospitais; unidades básicas de saúde; Serviço de Verificação de Óbitos (SVO) e Instituto Médico Legal (IML); serviços de transporte de pacientes (ambulâncias, SAMU Serviço de Atendimento Móvel de Urgência, Corpo de Bombeiros); cemitérios oficiais e não oficiais (locais de sepultamento); funerárias; delegacias de polícia; parteiras tradicionais; igrejas e farmácias. Foram investigados cartórios e hospitais de cada município da amostra e dos municípios vizinhos, onde ocorrem nascimentos e óbitos daquele município 29.

Para a comprovação da residência e do ano do falecimento (2008), os óbitos ou nascimentos encontrados na busca ativa sem registro de ocorrência nos cartórios ou nas unidades básicas de saúde foram confirmados nos domicílios, por meio de entrevista com algum membro da família do falecido. 
Os fatores de correção das estatísticas vitais entre os municípios foram calculados com base nos óbitos ou nascimentos adicionais captados na busca ativa após a confirmação dos eventos 28,29.

Depois de 2008, os resultados da pesquisa foram generalizados para que a metodologia fosse aplicada para todos os municípios brasileiros em outros anos, possibilitando estimar indicadores de mortalidade com o uso das informações vitais do Ministério da Saúde em todas as UF e regiões a partir do ano 20005.

Para a aplicação do procedimento de correção dos óbitos infantis no período 2000-2010, todos os municípios brasileiros foram classificados por nível de cobertura das informações de mortalidade, caracterizado pelo coeficiente de mortalidade padronizado por idade, sendo a população do Brasil, em cada ano do período, considerada como padrão. Os óbitos informados entre menores de um ano em todos os municípios brasileiros foram corrigidos pelos correspondentes fatores de correção obtidos na pesquisa de busca ativa de óbitos, de acordo com a categoria do coeficiente de mortalidade padronizada por idade $(<2 ; \geq 2 \mathrm{e}<3 ; \geq 3 \mathrm{e}<4 ; \geq 4 \mathrm{e}<5 ; \geq 5 \mathrm{e}<5,5 ; 5,5$ ou mais por mil habitantes) 4 .

Com esse método, os fatores de correção são mantidos constantes por categoria de coeficiente de mortalidade padronizada por idade, mas não por município. Isso é, se há uma melhora na cobertura das informações de mortalidade em um determinado município ao longo do período, é aplicado um fator de correção menor na medida em que ele passa para outra categoria de coeficiente de mortalidade padronizada por idade. O número corrigido de óbitos infantis, por UF e ano, é obtido pela soma dos óbitos corrigidos dos municípios daquela UF 4,5.

Analogamente, para a correção dos nascidos vivos no período 2000-2010, todos os municípios brasileiros foram classificados quanto à adequação das informações de nascidos vivos, estabelecida pela razão entre nascidos vivos informados e estimados. Após o agrupamento dos municípios pela razão de nascidos vivos informados e estimados $(<0,5 ; \geq 0,5 \mathrm{e}<0,6 ; \geq 0,6 \mathrm{e}<0,7 ; \geq 0,7 \mathrm{e}<0,8 ; \geq 0,8 \mathrm{e}$ $<0,9 ; \geq 0,9)$, os nascidos vivos foram corrigidos pelos fatores obtidos na busca ativa correspondente a cada categoria 4 .

De forma semelhante à correção dos dados de mortalidade, os fatores de correção para os nascidos vivos são estimados por categoria da razão entre nascidos vivos informados e estimados. Portanto, se há uma melhora na cobertura das informações de nascidos vivos em um determinado município ao longo do período, é aplicado um fator de correção menor à proporção que ele passa para outra categoria de razão de nascidos vivos informados e estimados. O número de nascidos vivos corrigido por UF é obtido pela soma dos nascidos vivos corrigidos dos municípios daquela UF 4,5.

\section{Mudança de paradigma no método de cálculo dos indicadores de mortalidade}

As pesquisas de busca ativa de óbitos e nascimentos mostraram-se essenciais para responder às questões iniciais relativas à possibilidade do uso dos dados de registro contínuo para a elaboração de indicadores fidedignos de mortalidade. A realização da pesquisa em uma amostra probabilística de municípios na Amazônia Legal e no Nordeste, estratificada por nível de adequação das informações vitais, possibilitou estimar fatores de correção em função da cobertura dos registros de óbitos e nascidos vivos do município e validar os critérios de adequação das informações propostos anteriormente 27.

A partir de 2008, o paradigma que norteava as publicações institucionais referentes à estimação das coberturas dos sistemas de informações vitais e da mortalidade infantil baseada em técnicas de mensuração indireta sofreu uma ruptura. Após a validação do método de correção das estatísticas vitais, todas as UF passaram a usar os dados vitais do Ministério da Saúde corrigidos por fatores condicionados ao nível de adequação das informações 27,28 .

A generalização da metodologia utilizada para a correção das estatísticas vitais pelo processo de busca ativa possibilitou estimar o coeficiente de mortalidade infantil no período 2000-2010, por UF e Grande Região 4,5. Observou-se um decréscimo acentuado do coeficiente de mortalidade infantil 4, diferentemente da tendência obtida mediante métodos demográficos indiretos no período 20002007 com base nas Pesquisas Nacionais por Amostra de Domicílios (PNAD), que apresentava progressiva diminuição do declínio após 2005, comprometendo a interpretação quanto ao desempenho das ações de saúde 30 . 
Em adição, a comparação da mortalidade infantil calculada com os dados vitais corrigidos e informados nas regiões Norte e Nordeste mostrou mudanças nas tendências de queda da taxa mortalidade infantil, com declínio anual mais intenso após a correção dos dados 5. Enfatiza-se, assim, a importância do uso de um método de estimação apropriado, uma vez que a aplicação de uma técnica inadequada pode alterar as tendências dos indicadores e induzir a interpretações e conclusões distintas em termos do progresso e alcance das metas 31. Um dos exemplos mais expressivos ao comparar as estimações da mortalidade infantil em 2008, antes e após as correções, foi o Estado de Alagoas, que migrou do segundo maior coeficiente para a décima primeira posição entre as UF, depois da correção dos dados informados 28 . A Figura 3 mostra a tendência do coeficiente de mortalidade infantil informado e corrigido no período 2000-2011.

Os ganhos referentes às informações vitais no Brasil, sobretudo nas duas últimas décadas, foram decorrentes dos investimentos do Ministério da Saúde na ampliação do acesso à assistência à saúde, associado às estratégias direcionadas à melhoria dos sistemas de informações. Entre essas, o suporte normativo ao processo de produção e análise dos dados; a constituição de pactos e a corresponsabilização dos níveis municipal, estadual e federal; a incorporação da busca ativa rotineira de óbitos e nascimentos não informados aos sistemas; iniciativas para reduzir a magnitude das causas mal definidas; a vigilância das mortes maternas, fetais, infantis e as sem definição da causa básica; a disponibilização de informações sobre óbitos e nascidos vivos por municípios no site do Departamento de Informática do SUS na Internet (http://www.datasus.gov.br); e a possibilidade de acompanhamento das informações por meio de um painel de monitoramento dos dados sobre mortalidade disponível na rede web (http://svs.aids.gov.br/dashboard/mortalidade/infantil.show.mtw) 32.

Em vista dos investimentos sistemáticos e contínuos, diversas avaliações vêm constatando os avanços na adequação das informações vitais 3,10,27,29. Um estudo abrangendo a década de 2000 mostrou que indicadores de cobertura, regularidade e qualidade das informações vitais em todas as regiões e grande parte das UF melhoraram, incontestavelmente, contribuindo para emergir novos desafios, sobretudo quanto ao aprimoramento da qualidade das informações 32 .

A situação do coeficiente geral de mortalidade padronizada, para os triênios 1996-1998 e 2011 2013, correspondendo aos períodos anterior e posterior às duas Pesquisas de Busca Ativa de eventos vitais, demonstram os grandes acréscimos na cobertura do SIM e SINASC, com redução de dados incompletos em todo país, mas também explicita que as coberturas permanecem desiguais, requerendo esforços adicionais e diferenciados entre as regiões e UF (Figuras 1 e 4).

\section{Estimativas subnacionais do coeficiente de mortalidade infantil}

Com o consenso brasileiro referente ao método de estimação do coeficiente de mortalidade infantil no âmbito das UF, surge o desafio da estimação para áreas geográficas subnacionais com menores contingentes populacionais, em particular os municípios, considerando a subenumeração de óbitos e nascidos vivos 33 .

No desenvolvimento da nova proposição, para garantir maior estabilidade aos indicadores diante da elevada proporção de municípios brasileiros com população inferior a 10 mil habitantes (45\%), as análises foram realizadas por triênio (2009-2011). Inicialmente, foram estimados fatores de correção de óbitos e nascidos vivos em todos os municípios com base em modelos de regressão com indicadores de cobertura das informações vitais. Após a correção inicial dos dados vitais, propôs-se procedimento para verificar se o número corrigido de óbitos infantis atingia o valor mínimo esperado de acordo com o porte populacional do município. Cabe destacar que em municípios muito pequenos o coeficiente de mortalidade infantil pode ser zero, e isto ser considerado como possível 33.

Os resultados dos procedimentos propostos se mostraram coerentes e apontaram que os fatores de correção estimados para os óbitos infantis foram insuficientes em poucos municípios (230), isto é, mesmo após a correção do número informado de óbitos infantis, a estimativa ficou abaixo do número mínimo esperado. Para esses municípios, o coeficiente de mortalidade infantil foi estimado por modelos de regressão utilizando-se como variáveis independentes características socioeconômicas e de acesso e utilização de serviços de saúde ${ }^{33}$. Nessa situação, embora as estimativas resultantes de modelos multivariados não substituam as realizadas diretamente quando os dados vitais são adequados, 
Figura 3

Coeficiente de mortalidade infantil antes e após correção. Brasil e regiões Norte e Nordeste, 2000-2011.

3a) Norte
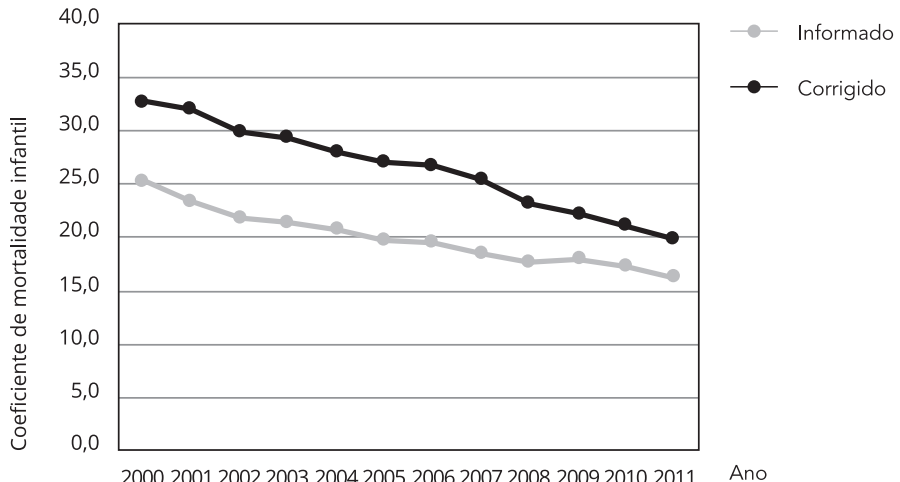

3b) Nordeste

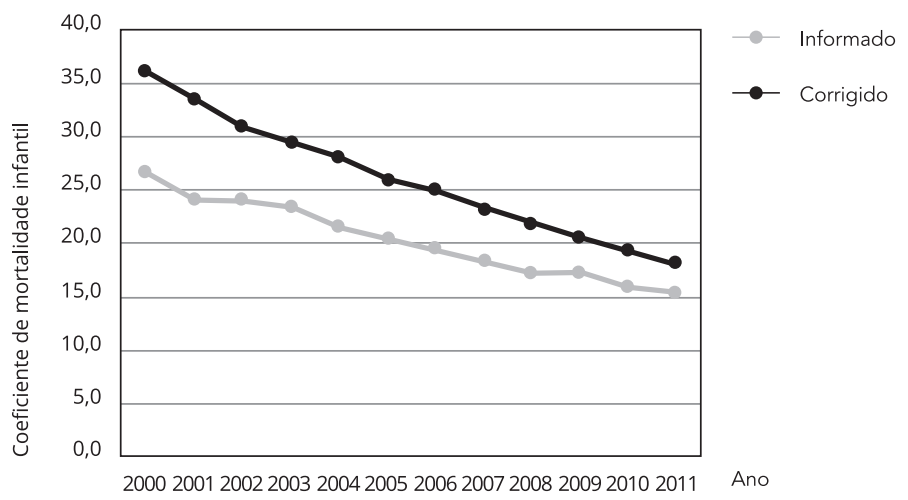

3c) Brasil

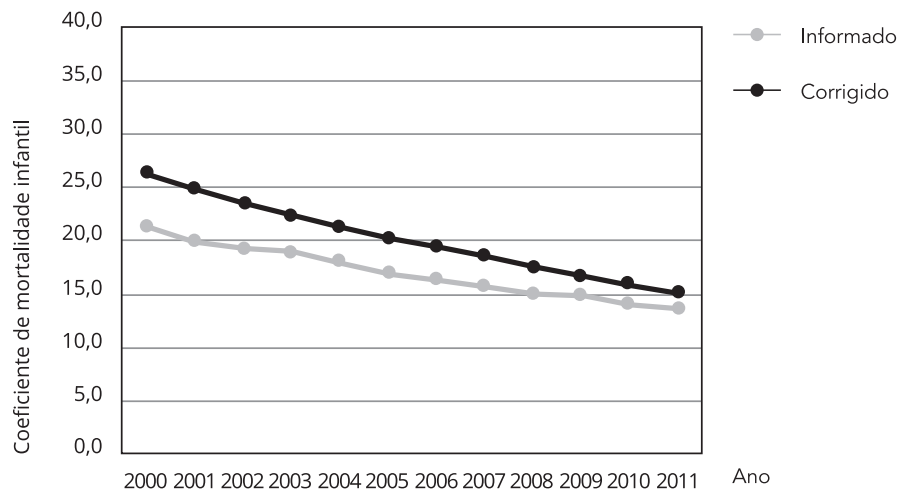


Figura 4

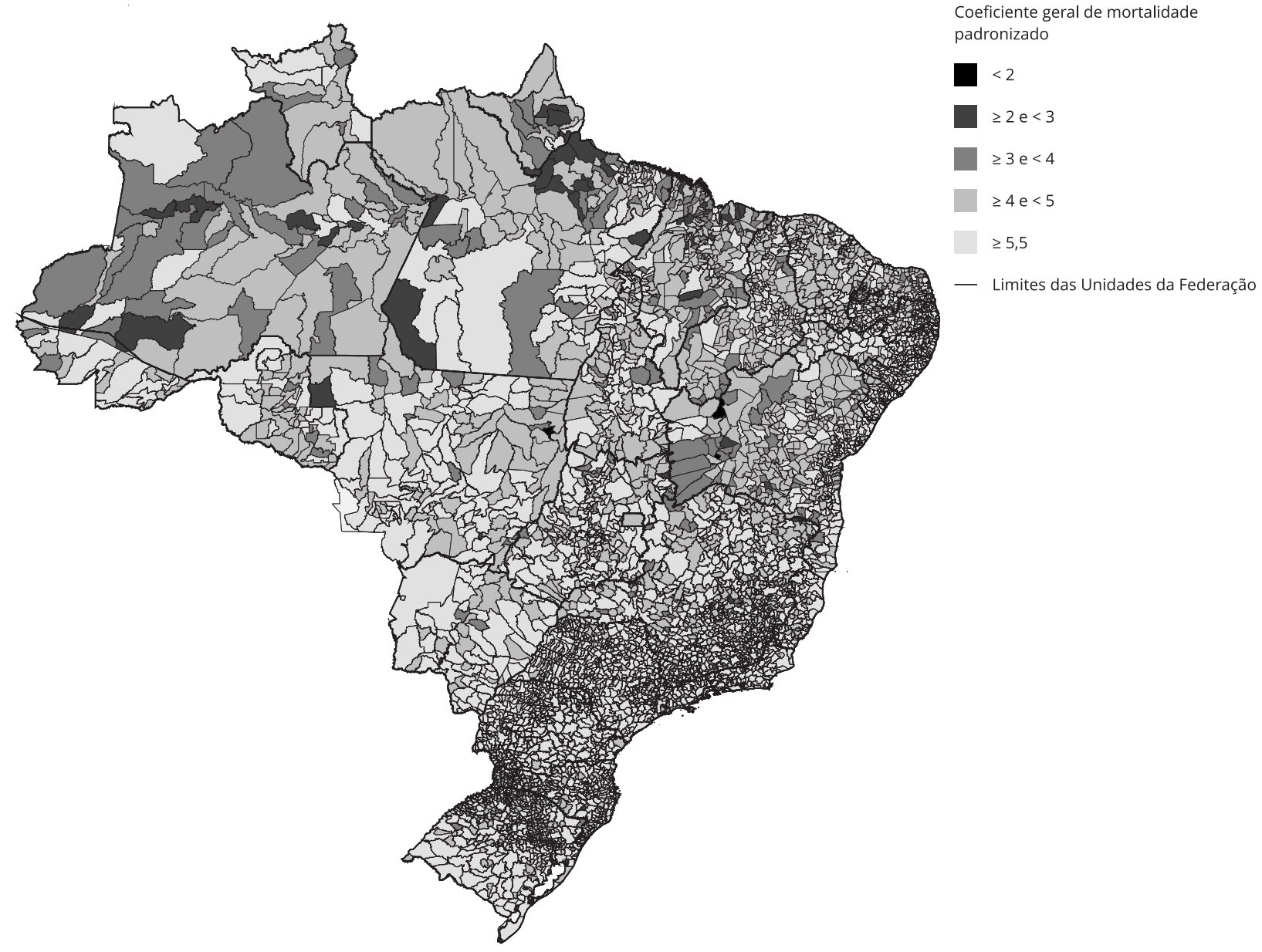

podem ser úteis para mostrar que em determinados municípios o número informado de óbitos infantis está bem abaixo do esperado, de acordo com as suas condições socioeconômicas e de saúde.

Apesar de mais um passo alcançado para valorar o uso dos dados diretos dos sistemas de informações, a permanência de 230 municípios brasileiros, cerca de $4 \%$ do total, ainda com registros inferiores ao número mínimo esperado sinaliza a necessidade de investimentos adicionais. Ao evidenciar dificuldades contextuais relacionadas à captação de eventos vitais e à estimação da mortalidade infantil, os achados contribuíram para o aprimoramento de políticas e estratégias de intervenção focadas para essas realidades, favorecendo a redução das iniquidades persistentes no país 34 .

Após a segunda busca ativa, a questão inicial sobre como estimar a mortalidade infantil no Brasil por meio das informações do Ministério da Saúde foi respondida de forma plenamente satisfatória. Entretanto, com a melhoria das informações, novas questões se descortinaram, como a estimação de fatores para corrigir os óbitos informados ao SIM por sexo e faixa de idade, e a estimação da mortalidade infantil em municípios pequenos com grande subenumeração dos óbitos infantis, em especial aqueles que se mantêm abaixo do mínimo esperado, mesmo após o uso dos fatores de correção. Igualmente importante é a questão da estimação de taxas específicas de mortalidade por causas de óbito, valorizando a investigação dos óbitos mal definidos e insuficientemente esclarecidos, como os códigos inespecíficos (garbage codes), e melhorando a acurácia da informação da causa de óbito 35. 
Pesquisas que contemplam parte dessas questões estão em andamento, como a realização da terceira edição da busca ativa, referente a óbitos ocorridos em 2012, que teve o objetivo principal de validar os fatores de correção dos dados vitais em uso, atualmente, nos municípios brasileiros, bem como estimar fatores para corrigir os óbitos informados ao SIM por sexo e faixa de idade incluindo amostra de municípios do Estado de Goiás, na Região Centro-oeste, e do norte de Minas Gerais, na Região Sudeste. Estudos sobre reclassificação das causas mal definidas também vêm sendo desenvolvidos, no sentido de aperfeiçoar as estimativas das taxas de mortalidade específicas por causa, além de projetos para avaliar a qualidade das informações 5,36,37. Pesquisas específicas sobre as informações do SINASC estão sendo, igualmente, realizadas para avaliar a qualidade de preenchimento dos dados por meio do relacionamento com dados coletados em inquéritos por amostragem, além de investigação dos óbitos perinatais de crianças viáveis, para distinguir nascidos vivos de natimortos por meio de registros hospitalares.

As pesquisas estão sendo realizadas por profissionais no âmbito da vigilância em saúde dos estados e municípios, contribuindo para a capacitação dos profissionais de saúde envolvidos e a incorporação dos conhecimentos e processos utilizados na rotina dos serviços.

\section{Colaboradores}

P. G. Frias contribui na concepção do artigo, participação nas pesquisas de busca ativa, análise dos dados, pesquisa bibliográfica e redação do manuscrito. C. L. Szwarcwald contribuiu na concepção do artigo, participação nas pesquisas de busca ativa, análise dos dados, pesquisa bibliográfica e redação do manuscrito. O. L. Morais Neto, M. C. Leal, J. J. Cortez-Escalante e P. R. B. Souza Junior tiveram participação nas pesquisas de busca ativa, análise dos dados e revisão do manuscrito. W. S. Almeida participou nas pesquisas de busca ativa, análise dos dados, pesquisa bibliográfica e revisão do manuscrito. J. B. Silva Junior participou nas pesquisas de busca ativa e revisão do manuscrito. Todos os autores revisaram e aprovaram a versão final do artigo.

\section{Agradecimentos}

Ao Ministério da Saúde pelo financiamento, e às Secretarias de Saúde dos estados e municípios participantes das várias edições das Pesquisas de Busca Ativa de Óbitos pelo apoio operacional ao trabalho de campo.

\section{Referências}

1. Barros FC, Matijasevich A, Requejo JH, Giugliani E, Maranhão AG, Monteiro CA, et al. Recent trends in maternal, newborn, and child health in Brazil: progress toward Millennium Development Goals 4 and 5. Am J Public Health 2010; 100:1877-89.

2. AbouZahr C, Savigny D, Mikkelsen L, Setel PW, Lozano R, Nichols E, et al. Civil registration and vital statistics: progress in the data revolution for counting and accountability. Lancet 2015; 386:1373-85.

3. Mello Jorge MHP, Laurenti R, Gotlieb SLD. Avaliação dos Sistemas de Informações em Saúde no Brasil. Cad Saúde Colet (Rio J.) 2010; 18:7-18.

4. Frias PG, Szwarcwald CL, Souza Jr PRB, Almeida WS, Lira PIC. Correção de informações vitais: estimação da mortalidade infantil, Brasil, 2000-2009. Rev Saúde Pública 2013; 47: 1048-58.

5. Szwarcwald CL, Frias PG, Souza Júnior PRB, Almeida WS, Morais Neto OL. Correction of vital statistics based on a proactive search of deaths and live births: evidence from a study of the North and Northeast regions of Brazil. Popul Health Metr 2014; 12:16.

6. Silva R. Child mortality estimation: consistency of under-five mortality rate estimates using full birth histories and summary birth histories. PLoS Med 2012; 9:e1001296.

7. Mathers C, Boerma T. Mortality measurement matters: improving data collection and estimation methods for child and adult mortality. PLoS Med 2010; 7:e1000265. 
8. Mahy M. Childhood mortality in the developing world: a review of evidence from the Demographic and Health Surveys. Calverton: ORC Macro; 2003. (DHS Comparative Reports, 4).

9. Murray CJ, Laakso T, Shibuya K, Hill K, Lopez AD. Can we achieve Millennium Development Goal 4? New analysis of country trends and forecasts of under-5 mortality to 2015 . Lancet 2007; 370:1040-54.

10. Szwarcwald CL. Strategies for improving the monitoring of vital events in Brazil. Int J Epidemiol 2008; 37:738-44.

11. Lima EEC, Queiroz BL. Evolution of the deaths registry system in Brazil: associations with changes in the mortality profile, under-registration of death counts, and ill-defined causes of death. Cad Saúde Pública 2014; 30:1721-30.

12. Viswanathan K, Becker S, Hansen PM, Kumar D, Kumar B, Niayesh H, et al. Infant and underfive mortality in Afghanistan: current estimates and limitations. Bull World Health Organ 2010; 88:576-83.

13. Murray CJ, Rajaratnam JK, Marcus J, Laakso T, Lopez AD. What can we conclude from death registration? Improved methods for evaluating completeness. PLoS Med 2010; 7:e1000262.

14. Frias PG, Szwarcwald CL, Lira PIC. Estimação da mortalidade infantil no contexto de descentralização do Sistema Único de Saúde (SUS). Rev Bras Saúde Matern Infant 2011; 11:463-70.

15. Secretaria de Saúde. Mortalidade infantil em Pernambuco (1980-1991): geografia, magnitude e significados. Recife: Secretaria de Saúde; 1996.

16. Frias PG, Vidal SA, Pereira PMH, Lira PIC, Vanderlei LC. Avaliação da notificação de óbitos infantis ao Sistema de Informações sobre Mortalidade: um estudo de caso. Rev Bras Saúde Matern Infant 2005; 5 Suppl 1:S43-51.

17. Simões CC. Estimativas da mortalidade infantil por microrregiões e municípios. Brasília: Ministério da Saúde; 1999.

18. Szwarcwald CL, leal MC, Andrade CLT, Souza Jr. PRB. Estimação da mortalidade infantil no Brasil: o que dizem as informações sobre óbitos e nascimentos do Ministério da Saúde? Cad Saúde Pública 2002; 18:1725-36.

19. Frias PG, Pereira PMH, Andrade CLT, Lira PIC, Szwarcwald CL. Avaliação da adequação das informações de mortalidade e nascidos vivos no Estado de Pernambuco, Brasil. Cad Saúde Pública 2010; 26:671-81.

20. Frias PG, Pereira PMH, Andrade CLT, Szwarcwald CL. Sistema de Informações sobre Mortalidade: estudo de caso em municípios com precariedade dos dados. Cad Saúde Pública 2008; 24:2257-66.

21. Nations MK. Infant death and interpretive violence in Northeast Brazil: taking bereaved Cearense mothers' narratives to heart. Cad Saúde Pública 2008; 24:2239-48.

22. Nations MK, Rebhun LA. Angels with wet wings won't fly: maternal sentiment in Brazil and the image of neglect. Cult Med Psychiatry 1988; 12:141-200.
23. Campos D, Hadad SC, Abreu DMX, Cherchiglia ML, França E. Sistema de Informações sobre Mortalidade em municípios de pequeno porte de Minas Gerais: concepções dos profissionais de saúde. Ciênc Saúde Coletiva 2013; 18:1473-82.

24. Ministério da Saúde. Importância dos sistemas de informações sobre mortalidade e nascidos vivos para os profissionais do Programa Saúde da Família. Brasília: Fundação Nacional de Saúde; 2001

25. Ministério da Saúde. Portaria no 116, de 11 de fevereiro de 2009. Regulamenta a coleta de dados, fluxo e periodicidade de envio das informações sobre óbitos e nascidos vivos para os Sistemas de Informações em Saúde sob gestão da Secretaria de Vigilância em Saúde. Diário Oficial da União 2009; 12 fev.

26. Andrade CLT, Szwarcwald CL. Desigualdades sócio-espaciais da adequação das informações de nascimentos e óbitos do Ministério da Saúde, Brasil, 2000-2002. Cad Saúde Pública 2007; 23:1207-16.

27. Szwarcwald CL, Morais Neto OL, Escalante JJC, Souza Jr. PRB, Frias PG, Lima RB, et al. Busca ativa de óbitos e nascimentos no Nordeste e na Amazônia Legal: estimação das coberturas do SIM e do SINASC nos municípios brasileiros. In: Ministério da Saúde, organizador. Saúde Brasil 2010: uma análise da situação de saúde e de evidências selecionadas de impacto de ações de vigilância em saúde. Brasília: Ministério da Saúde; 2011. p. 79-98.

28. Szwarcwald CL, Morais Neto OL, Frias PG, Viola RC. Busca ativa de óbitos e nascimentos no Nordeste e na Amazônia Legal: estimação da mortalidade infantil nos municípios brasileiros. In: Ministério da Saúde, organizador. Saúde Brasil 2010: uma análise da situação de saúde e de evidências selecionadas de impacto de ações de vigilância em saúde. Brasília: Ministério da Saúde; 2011. p. 99-116.

29. Figueiroa BQ, Vanderlei LCM, Frias PG, Carvalho PI, Szwarcwald CL. Análise da cobertura do Sistema de Informações sobre Mortalidade em Olinda, Pernambuco, Brasil. Cad Saúde Pública 2013; 29:475-84.

30. Victora CG, Aquino EM, Leal MC, Monteiro CA, Barros FC, Szwarcwald CL. Maternal and child health in Brazil: progress and challenges. Lancet 2011; 377:1863-76.

31. Alkema L, You D. Child mortality estimation: a comparison of UN IGME and IHME estimates of levels and trends in under-five mortality rates and deaths. PLoS Med 2012; 9:e1001288.

32. Frias PG, Szwarcwald CL, Lira PIC. Avaliação dos sistemas de informações sobre nascidos vivos e óbitos no Brasil na década de 2000. Cad Saúde Pública 2014; 30:2068-80.

33. fantil nos municípios brasileiros: uma proposta de método de estimação. Rev Bras Saúde Matern Infant 2014; 14:331-42. 
34. Phillips DE, AbouZahr C, Lopez AD, Mikkelsen L, Savigny D, Lozano R, et al. Are well functioning civil registration and vital statistics systems associated with better health outcomes? Counting births and deaths 2. Series. Lancet 2015; 386:1386-94.

35. Schmidt MI, Duncan BB, Ishitani L, Conceição Franco G, Abreu DM, Lana GC, et al. Trends in mortality due to diabetes in Brazil, 1996-2011. Diabetol Metab Syndr 2015; 7:109.

\section{Abstract}

The article addresses Brazil's historical development in the use of vital data, incorporating procedures for the evaluation of such data and research with active search of births and deaths, resulting in the proposal of methods for calculating birth and mortality indicators through the use of continuous records. In addition to research to capture vital events from the years 2000 and 2008, the article presents procedures for the correction of events reported to the information systems and the paradigm shift in the method for calculating mortality indicators, resulting from such initiatives. The study also features advances in the adequacy of information on deaths and live births in Brazil, changes in the estimates on infant mortality resulting from the proposed methods, and the challenge of estimating the indicator for subnational geographic areas with lower population contingents, mostly consisting of municipalities (counties) with low and irregular data coverage.

Vital Statistics; Information Systems; Mortality Registries; Birth Registration
36. França E, Teixeira R, Ishitani L, Duncan BB, Cortez-Escalante JJ, Morais Neto OL, et al. Causas mal definidas de óbito no Brasil: método de redistribuição baseado na investigação do óbito. Rev Saúde Pública 2014; 48:671-81.

37. Mikkelsen L, Phillips DE, AbouZahr C, Setel PW, Savigny D, Lozano R, et al. A global assessment of civil registration and vital statistics systems: monitoring data quality and progress. Lancet 2015; 386:1395-406.

\section{Resumen}

El artículo presenta la construcción histórica brasileña sobre la utilización de la información vital, incorporando procedimientos de evaluación de la información e investigaciones de búsqueda activa de nacimientos y óbitos, que resultaron en la propuesta de métodos para el cálculo de indicadores de natalidad y mortalidad, mediante el uso de registros continuos. Además de las investigaciones para captar eventos vitales, referentes a los años de 2000 y 2008, se presentan los procedimientos para la corrección de los eventos informados en los sistemas de información y el cambio de paradigma en el método del cálculo de los indicadores de mortalidad, derivado de estas iniciativas. Asimismo, se destacan los avances en la adecuación de la información sobre óbitos y nacidos vivos en Brasil, las alteraciones en las estimativas de la mortalidad infantil, derivadas de la propuesta de métodos, además del desafío de estimar el indicador para áreas geográficas subnacionales, con menores contingentes poblacionales, que en su mayoría están compuestas por municipios con baja cobertura y regularidad de datos.

Estadísticas Vitales; Sistemas de Información; Registros de Mortalidad; Registro de Nacimiento
Recebido em 08/Jan/2016

Versão final reapresentada em 03/Mai/2016

Aprovado em 02/Jun/2016 